\title{
The German version of the Child Behavior Checklist I.5-5 to identify children with a risk of autism spectrum disorder
}

\author{
Katharina Limberg', Karolin Gruber ${ }^{2}$ and Michele Noterdaeme'
}

\begin{abstract}
A long delay between the first registered symptoms of autism spectrum disorder and a final diagnosis has been reported. The reasons for this are the spare use of specialized autism instruments, missing clinical expertise, and the late referral to specialized centers in primary care. Previous studies recommending the Child Behavior Checklist I.5-5 for screening have requested additional research. A total of 183 children aged 25-7I months participated in this study. The Child Behavior Checklist scales of 80 children with autism spectrum disorder were compared with 103 children diagnosed with other psychiatric disorders. In the logistic regression analysis, the Withdrawn and Pervasive Developmental Problems Child Behavior Checklist scales with a significant predictive value of risk for an autism spectrum disorder diagnosis were identified. The optimal cutoff points $T=64.5$ on the Pervasive Developmental Problems scale (area under the curve $=0.78 \mathrm{I}$, sensitivity $=0.83$, specificity $=0.60$, positive predictive value $=0.62$, negative predictive value $=0.82$, odds ratio $=7$ ) and $T=60.5$ on the Withdrawn scale (area under the curve $=0.809$, sensitivity $=0.88$, specificity $=0.63$, positive predictive value $=0.65$, negative predictive value $=0.87$, odds ratio $=12$ ) were evaluated in the receiver operating characteristics analysis. The present study confirms the utility of the German version of the Child Behavior Checklist I.5-5 as a level I screening tool to identify children with a risk of autism spectrum disorder; however, a risk of overidentifying should be considered. The Child Behavior Checklist I.5-5 can complement the pediatric examination as a quick and cost-effective questionnaire.
\end{abstract}

\section{Keywords}

autism spectrum disorder, Child Behavior Checklist I.5-5, early detection, preschool children, screening

\section{Introduction}

Autism spectrum disorder (ASD) is classified in the Diagnostic and Statistical Manual of Mental Disorders (DSM-5) as a heterogeneous group of neurodevelopmental disorders characterized by persistent deficits in social communication and interaction accompanied by restricted, repetitive patterns of behavior, interests, or activities. In addition to these core domains, the diagnostic criteria require the appearance of symptoms in early childhood, which cause impairments of everyday functioning and must not be explained by intellectual disability or global developmental retardation. In recent years, a significant increase in the prevalence can be observed. Currently, the prevalence rate for ASD is estimated at about 1\% (American Psychiatric Association (APA), 2013). Until now, definitive reasons for this increase are not specified. Modified diagnosis criteria and the growing awareness of ASD are discussed, while a real increase of the prevalence rate is negated (Freitag and Petermann, 2014). This is one key factor why diagnostic assessment of ASD becomes more and more relevant in clinical routine and specialist literature.

Because of the wide variability of behavioral phenotypes and in severity, an accurate ASD diagnostic is difficult. The gold standards for diagnosing ASD have been utilized (National Collaborating Centre for Women's and Children's Health (UK), 2011): the second edition of the

Josefinum, Germany

2Ludwig-Maximilians-Universität München, Germany

Corresponding author:

Katharina Limberg, Department of Child and Adolescent Psychiatry and Psychotherapy, Josefinum, Kapellenstrasse 30, 86I54 Augsburg, Germany.

Email: KatharinaLimberg@aol.com 
Autism Diagnostic Observation Schedule (ADOS-2; Lord et al., 2012) and the Autism Diagnostic Interview-Revised (ADI-R; Rutter et al., 2003). The correct use of these specialized autism instruments requires extensive training and knowledge, as well as clinical experience with ASD. These are a few of the reasons that the specialized assessment instruments are used by experts only in ASD diagnostic and usually not in primary care and educational settings.

The majority of the first reported symptoms, such as language, communication, and social interaction problems, are typical for ASD. They are already registered during the second year of life. Even if parents become concerned about the development of their child quite early, a definitive diagnosis in Germany is made at a mean age of 76 months, often not until the children enter elementary school. This means that there is a long delay, on average by 61 months, between initial parental concern and the time when a diagnosis is made. Some precious years pass by when children could benefit from an early intervention. Parents suffer from anxiety and experience stress during this period. In a German study, Noterdaeme and Hutzelmeyer-Nickels (2010) could find that there was no significant reduction in the age at diagnosis between 1998 and 2007. One reason for the delay is the lack of clinical expertise with ASD in primary care and the associated late referral to a specialized center. In addition, there are only a few specialized centers with experience and established expertise in ASD (Noterdaeme and Hutzelmeyer-Nickels, 2010).

The role of the pediatrician becomes increasingly important in reducing this gap. In Germany a pediatrician sees children routinely during the medical checkups, nine times in the first 6 years of life (U1-U9). Furthermore, a pediatrician is likely the first contact person and often the first professional who sees a child with ASD. For this reason, it is necessary to introduce a level 1 screening instrument for non-specialized professionals in primary care to identify children with a risk of ASD. Currently, there are more than 20 screening tools available in Europe. Only in Spain is the Modified-Checklist for Autism in Toddlers (M-CHAT) used in routine screening procedures, while in most other countries the screening instruments are utilized only by ASD specialists and are not part of routine practice. Because of the variety of health care and government policy in various countries, a standardization of the screening procedure in Europe is not possible (García-Primo et al., 2014). A solution could be a broadband behavior rating scale as a screener, a cost-effective and expeditious method that is already widespread in primary settings and requires less specialized knowledge of ASD for evaluation. All these requirements meet the Child Behavior Checklist (CBCL), one of the most widely used parent report checklists (Achenbach and Rescorla, 2000). The CBCL examines a diversity of behavior and emotional problems and even includes the DSM-oriented scale
Pervasive Developmental Problems (PDP), which represents some ASD-specific items. In addition, it shows appropriate reliability and validity (Achenbach and Rescorla, 2000; Ivanova et al., 2010; Pandolfi et al., 2009).

A few previous studies have examined the CBCL 1.5-5 as a screening instrument for young children with ASD: for example, Havdahl et al. (2015), Myers et al. (2014), and Sikora et al. (2008) for the United States; Rescorla et al. (2014) for Korea; Narzisi et al. (2013) and Muratori et al. (2011) for Italy. To our knowledge, Rescorla (1988) first tested the ability of the CBCL $1.5-5$ for identification of autistic preschoolers. Most of the studies recommend the CBCL 1.5-5 as an ASD screener. Nevertheless, current research is rare, and all of the above-mentioned studies require additional research to analyze the applicability of the CBCL $1.5-5$.

The aim of this study is to examine whether the CBCL $1.5-5$ can be used in Germany as a level 1 screening instrument to identify children with a risk of ASD. In the process, significant CBCL scales should be detected and cutoff points calculated, which indicate an actual risk of ASD. Previous studies (as mentioned above) describe a good ability of the CBCL $1.5-5$ to distinguish between children with ASD from typically developing children. We expect the same result from our research. Contrary to this, it is especially hard to differentiate between ASD and other clinically referred children in primary settings. For this reason, the main focus of this study is the identification of CBCL scales to discriminate children with ASD from children with other psychiatric disorders (OPDs). Following the CBCL 1.5-5 could be able to support non-specialized professionals (e.g. pediatricians) in deciding whether a recommendation for a more in-depth and specialized ASD assessment is needed. Therefore, it is possible to accelerate a precise ASD diagnosis and early intervention, which is known to be crucial for normalized patterns of brain activity and consequently improvements in social behavior. An enhancement of the long-term outcome is thereby possible (Dawson et al., 2012). Speeding up an ASD diagnosis is also associated with reducing parents stress and diagnostic uncertainty of the disorder (Wiberg et al., 2007).

\section{Method}

\section{Participants}

A total number of 183 children aged $25-71$ months (126 males, 57 females, mean age 53.8 months, standard deviation $(\mathrm{SD})=11.7)$ participated in the study. The experimental group included 80 children diagnosed with ASD (infantile autism (F 84.0) and asperger syndrome (F 84.5); 60 males, 20 females, mean age 53.2 months, $\mathrm{SD}=10.7$, range 25-71 months). The control group consisted of 103 children (66 males, 37 females, mean age 54.4 months, $\mathrm{SD}=12.5$, range $25-71$ months), all with a diagnosis of 
Table I. Diagnoses of the control group (OPD).

\begin{tabular}{lr}
\hline & $\mathrm{N}$ \\
\hline Reaction to severe stress and adjustment & 21 \\
disorders (F4) & 21 \\
Adjustment disorders (F43) & 125 \\
Developmental disabilities (F8) & 85 \\
Speech and language (F80) & $3 \mathrm{I}$ \\
Motor functions (F82) & 2 \\
Combined (F83) & $\mathrm{I}$ \\
Unspecified (F89) & 37 \\
Behavioral and emotional disorders (F9) & $2 \mathrm{I}$ \\
Attention-deficit hyperactivity disorders (F90) & 4 \\
Conduct disorders (F9I) & 2 \\
Combined (F92) & 3 \\
Emotional disorders (F93) & $\mathrm{I}$ \\
Disorder of social functioning (F94) & 6 \\
Other (F98) & $\mathrm{I}$ \\
\hline Intellectual disabilities (F7)
\end{tabular}

OPD: other psychiatric disorder.

OPDs. The diagnoses of the sample are adjustment disorders, developmental disabilities (except pervasive developmental disorders), behavioral and emotional disorders, and intellectual disabilities. Many of the children have more than one diagnosis (for details see Table 1). All children were recruited from and diagnosed at the Department of Child and Adolescent Psychiatry and Psychotherapy and the Interdisciplinary Early Intervention Centre at Josefinum Hospital in Augsburg, Germany, between February 2013 and February 2014.

They were examined by an experienced child psychiatrist (last author) and diagnosed on the basis of International Classification of Diseases (ICD)-10 criteria. The diagnosis of an ASD was made by an expert in autism. During the diagnostic process, the ADOS-Generic and ADI-R were used. In the control group (OPD), a diagnosis of ASD or another pervasive developmental disorder was strictly excluded.

\section{Measures}

The CBCL 1.5-5 (Achenbach and Rescorla, 2000) is a standardized parent questionnaire of behavior problems for children from 1.5 to 5 years of age. The respondents rate 99 specific problem items ( 0 for not true, 1 for somewhat or sometimes true, 2 for very true or often true) and describe the open-ended item 100 any additional problem, based on the previous 2 months. The form is filled out in about $10 \mathrm{~min}$ and analyzed by computerized scoring. The result constitutes a syndrome, DSM-oriented and summary profile. There are seven syndrome scales: Emotionally Reactive, Anxious/Depressed, Somatic Complaints, Withdrawn, Sleep Problems, Attention Problems, and Aggressive Behavior. Each represents items that tend to co-occur. The five DSM-oriented scales (Affective Problems, Anxiety Problems, PDP, Attention Deficit/ Hyperactivity Problems, and Oppositional Defiant Problems) are based on the Diagnostic and Statistical Manual of Mental Disorders (4th ed.; DSM-IV) of the APA. The summary profile consists of an Internalizing, Externalizing, and Total Problems scale. The four syndrome scales (Emotionally Reactive, Anxious/Depressed, Somatic Complaints, Withdrawn) are grouped under the rubric Internalizing and the two scales Attention Problems and Aggressive Behavior under the heading Externalizing. The scales Sleep and Stress Problems are not assigned to either of them. The Total Problems scale represents the highest level in the hierarchy and includes the sum of all 100 items. A T score $\geqslant 70$ at the syndrome and DSMoriented scales and $\geqslant 64$ on the summary scales indicates the clinical range. T-scores in the borderline range (65-69 on syndrome and DSM-oriented scales, 60-63 on Internalizing, Externalizing, and Total Problems scales) are high enough to be concerned; scores below are in the normal range (Achenbach and Rescorla, 2000).

\section{Procedures}

Based on the manual (Achenbach and Rescorla, 2000), the CBCL 1.5-5 was filled out by parents and others who see children in family settings. The questionnaires were computer-scored by a software for Achenbach System of Empirically Based Assessment (ASEBA) forms. The forms with more than eight blank items were excluded from the study. If the respondent circled two scores (1 and 2) or one was marked unclear, a score of 1 was used (for further information, see Achenbach and Rescorla, 2000: 136).

\section{Data analyses}

All scales of the CBCL 1.5-5 and the group characteristics, age and gender were tested for normal distribution using the Kolmogorov-Smirnov test. None of them showed normality. Comparing the experimental group with the control group on age, the Mann-Whitney U test was used. The chi-square test examined the difference in gender between the two groups.

The logistic regression analysis with $\operatorname{Exp}(\mathrm{B})$ was used to identify significant CBCL scales distinguishing ASD from OPD. For that, different logistic regression models were constructed. The dependent variable (ASD, yes or no) was invariant. The independent variable consisted of different CBCL scales and differentiated between the models. In model 1, the independent variable was the Total Problems scale; in model 2, the independent variables were the Internalizing and Externalizing Problems scales; in model 3, the independent variables were all syndrome scales; in model 4 , the independent variables were all 


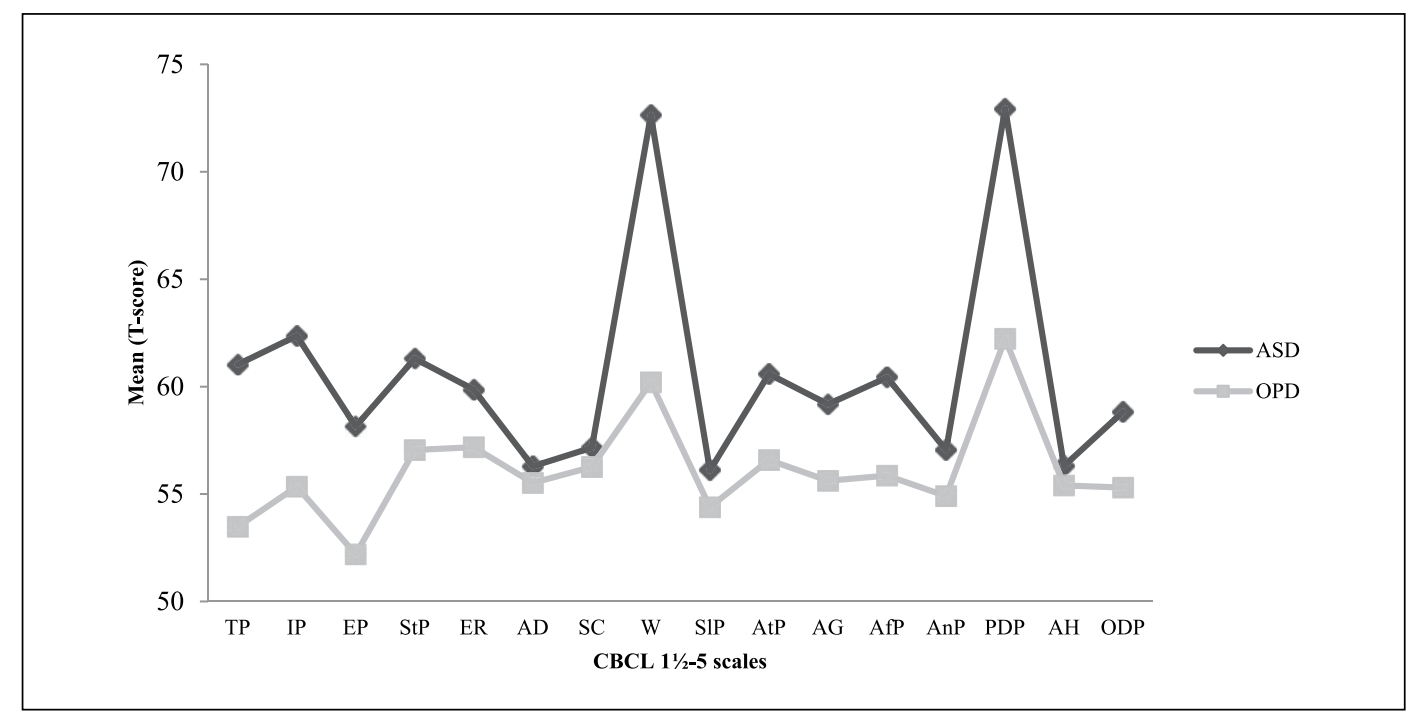

Figure I. Mean values of $\mathrm{CBCL}$ T-scores.

TP: Total Problems; IP: Internalizing Problems; EP: Externalizing Problems; StP: Stress Problems; ER: Emotionally Reactive; AD: Anxious/Depressed; SC: Somatic Complaints; W: Withdrawn; SIP: Sleep Problems; AtP: Attention Problems; AG: Aggressive Behavior; AfP: Affective Problems; AnP: Anxiety Problems; PDP: Pervasive Developmental Problems; AH: Attention Deficit/Hyperactivity Problems; ODP: Oppositional Defiant Problems.

DSM-oriented scales; and in model 5, the independent variable was the Stress Problems scale.

In the following receiver operating characteristics (ROC) analysis, the CBCL scales with a predictive value for an ASD diagnosis, identified in the logistic regression analysis, were examined to detect their optimal cutoff points. The cutoff point describes the optimal compromise between sensitivity (true positive rate) and specificity (true negative rate), with the intention to discriminate between children with ASD and OPD.

To evaluate the accuracy of the diagnostic instrument, the area under the curve (AUC) was used. Based on criteria of Swets (1988), the AUC value was interpreted as low diagnostic accuracy for AUC $<0.7$, moderate diagnostic accuracy for AUC range $0.7-0.9$ and high diagnostic accuracy for AUC $>0.9$.

For each optimal cutoff point, the positive predictive value (PPV; proportion of a positive test result that is true positive), negative predictive value (NPV; proportion of a negative test result that is true negative), and odds ratio (OR) were calculated.

To adjust the level of significance related to the problem of multiple testing, the Bonferroni correction was used, with the result of $p<0.007$. The data were analyzed with the assistance of SPSS version 20.

\section{Results}

\section{Preliminary analyses}

A total of 183 children with the mean age of 53.8 months $(\mathrm{SD}=11.7)$ participated in the study. The children with an ASD diagnosis (mean age 53.2 months, $\mathrm{SD}=10.7$ ) were younger than the children with an OPD diagnosis (mean age 54.4 months, $\mathrm{SD}=12.5$ ). The Mann-Whitney $\mathrm{U}$ test was used to test the influence of age and showed no significant difference among the two groups $(\mathrm{p}=0.344)$.

Group differences on gender were calculated with the chi-square test. The percentage of males in the ASD group (75\% males, $25 \%$ females) were higher than in the OPD group (64.1\% males, $35.9 \%$ females), but the difference was statistically not significant (chi-square $=2.505$, $\mathrm{p}=0.114)$.

\section{Clinical characteristics}

On first examination the comparison of means showed that the children with ASD diagnosis presented higher mean values with varying extent on all CBCL scales than the children with OPD diagnosis (see Figure 1).

In the logistic regression analysis with $\operatorname{Exp}(\mathrm{B})$, comparing the two groups, the CBCL scales Withdrawn $(\operatorname{Exp}(\mathrm{B})=1.14,95 \%$ confidence interval $(\mathrm{CI}) 1.10-1.19)$, PDP $(\operatorname{Exp}(\mathrm{B})=1.14 ; 95 \%$ CI $1.09-1.20)$, and Total Problems $(\operatorname{Exp}(\mathrm{B})=1.06 ; 95 \%$ CI $1.03-1.09)$ were detected as scales with a significant predictive value of a risk for an ASD diagnosis $(p<0.001)$. The results of the logistic regression analysis are represented in Table 2.

\section{ROC analyses}

In the ROC analysis, the optimal cutoff points for the predictor CBCL scales Withdrawn, PDP, and Total Problems were identified. To discriminate children with ASD from children with OPD, the optimal cutoff point on the Withdrawn scale was determined at a score of $\mathrm{T}=60.5$ 
Table 2. Mean values and logistic regression analysis with $\operatorname{Exp}(B)$ on $C B C L$ I.5-5 T-scores.

\begin{tabular}{|c|c|c|c|c|c|}
\hline \multirow[t]{3}{*}{$\mathrm{CBCL}$ scales } & \multirow{3}{*}{$\begin{array}{l}\text { ASD } \\
(N=80)\end{array}$} & \multirow{3}{*}{$\begin{array}{l}\text { OPD } \\
(N=103)\end{array}$} & \multicolumn{3}{|c|}{ Logistic regression with $\operatorname{Exp}(\mathrm{B})$ and $95 \% \mathrm{Cl}$} \\
\hline & & & \multicolumn{3}{|c|}{ ASD versus OPD } \\
\hline & & & $\mathrm{P}$ & $\operatorname{Exp}(B)$ & $95 \% \mathrm{Cl}$ \\
\hline Total Problems & 61.03 & 53.48 & $<0.001$ & 1.06 & $1.03-1.09$ \\
\hline Internalizing Problems & 62.36 & 55.35 & 0.010 & 1.06 & $1.01-1.10$ \\
\hline Externalizing Problems & 58.15 & 52.20 & 0.572 & 1.01 & $0.97-1.05$ \\
\hline Stress Problems & 61.31 & 57.05 & 0.080 & 1.06 & $0.99-1.13$ \\
\hline Emotionally Reactive & 59.86 & 57.18 & 0.194 & 0.95 & $0.88-1.03$ \\
\hline Anxious/Depressed & 56.29 & 55.52 & 0.347 & 0.97 & $0.90-1.04$ \\
\hline Somatic Complaints & 57.16 & 56.26 & 0.540 & 0.98 & $0.93-1.04$ \\
\hline Withdrawn & 72.64 & 60.20 & $<0.001$ & 1.14 & $1.10-1.19$ \\
\hline Sleep Problems & 56.11 & 54.39 & 0.927 & 1.00 & $0.95-1.06$ \\
\hline Attention Problems & 60.59 & 56.59 & 0.227 & 1.04 & $0.98-1.09$ \\
\hline Aggressive Behavior & 59.18 & 55.62 & 0.662 & 1.01 & $0.96-1.08$ \\
\hline Affective Problems & 60.43 & 55.86 & 0.771 & 1.01 & $0.95-1.07$ \\
\hline Anxiety Problems & 57.05 & 54.91 & 0.054 & 0.95 & $0.89-1.00$ \\
\hline Pervasive Developmental Problems & 72.94 & 62.23 & $<0.001$ & 1.14 & $1.09-1.20$ \\
\hline Attention Deficit/Hyperactivity Problems & 56.31 & 55.40 & 0.124 & 0.95 & $0.89-1.01$ \\
\hline Oppositional Defiant Problems & 58.83 & 55.30 & 0.429 & 1.02 & $0.97-1.08$ \\
\hline
\end{tabular}

Exp(B): Odds; CBCL: Child Behavior Checklist; Cl: confidence interval; ASD: autism spectrum disorder; OPD: other psychiatric disorder.

( sensitivity $=0.88$, specificity $=0.63$ ). The ROC curve shown in Figure 2 (a graphical plot of 1-specificity against sensitivity) represented for this cutoff point an AUC of 0.809 and indicated in that way a moderate diagnostic accuracy. A PPV of 0.65 , an NPV of 0.87 , and an OR of 12 were calculated. For the CBCL scale PDP, the optimal compromise between sensitivity $(0.83)$ and specificity (0.60) to discriminate the ASD group from the OPD group was made at a cutoff point of $\mathrm{T}=64.5$. Using this cutoff point, an AUC with a moderate diagnostic accuracy of 0.781 was indicated. The calculation showed a PPV of 0.62 , an NPV of 0.82 , and an OR of 7 . The best cutoff point for the Total Problems scale discriminating the two groups was at a score of $\mathrm{T}=52.5$ (sensitivity $=0.80$, specificity $=0.50$ ). An AUC of 0.686 on this cutoff point showed only a low diagnostic accuracy. The examination of the values for PPV (0.55), NPV (0.76), and OR (4) resulted in much lower values than on the other scales (see Table 3 ).

\section{Discussion}

Screening tests must meet strict criteria to be effective. Among other things, they have to be brief, standardized, objectively scored, and inexpensive (Meisels, 1989). The CBCL 1.5-5 meets all these requirements.

As expected, the results of the mean comparison show that children with an ASD diagnosis presented higher mean values on all CBCL scales than the children with an OPD diagnosis. However, only three scales-PDP, Withdrawn, and Total Problems - indicate a significant predictive value of a risk for an ASD diagnosis.

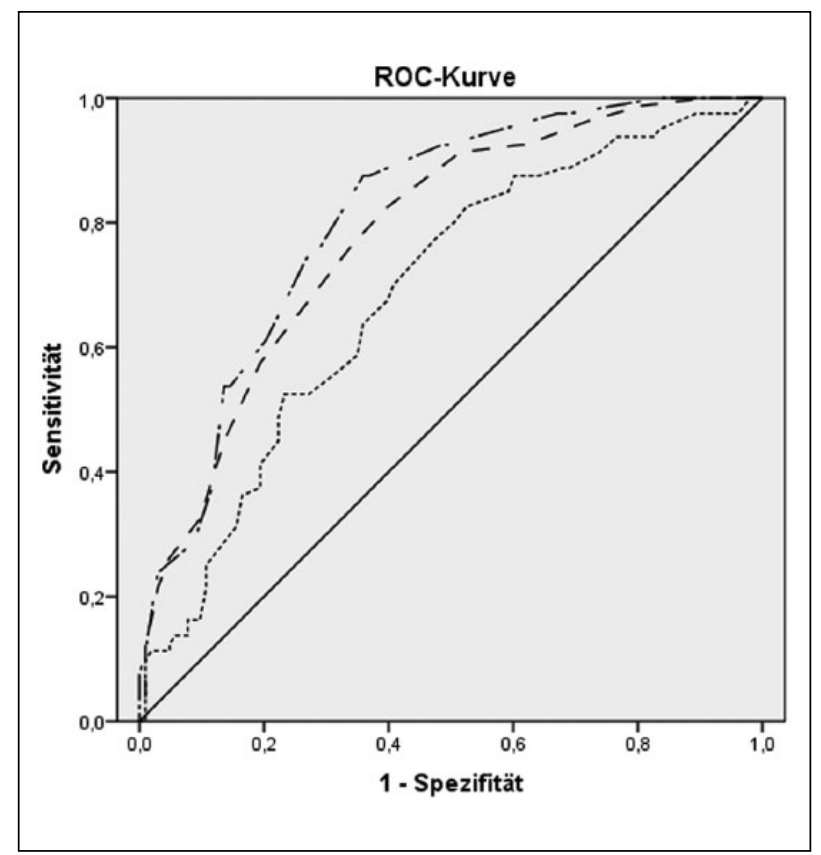

Figure 2. ROC curve for PDP $(T=64.5)$, Withdrawn $(T=60.5)$, and Total Problems $(T=52.5)$.

- - -Withdrawn; --.- PDP; ....... Total Problems; _ _ reference line.

For the cutoff point determination, we looked for the optimal compromise between sensitivity and specificity. According to Meisels (1989), criteria for screening tests sensitivity and specificity should be no less than $80 \%$. Specific attention was paid to a high sensitivity, which 
Table 3. Sensitivity, Specificity, PPV, NPV, AUC, diagnostic accuracy and OR at the optimal cutoff points on the Total Problems, Withdrawn, and PDP scales.

\begin{tabular}{llll}
\hline & Total Problems (cutoff T=52.5) & Withdrawn (cutoff T=60.5) & PDP (cutoff T=64.5) \\
\hline Sensitivity & $80 \%$ & $88 \%$ & $83 \%$ \\
Specificity & $50 \%$ & $63 \%$ & $60 \%$ \\
PPV & $55 \%$ & $65 \%$ & $62 \%$ \\
NPV & $76 \%$ & $87 \%$ & $82 \%$ \\
AUC & 0.686 & 0.809 & 0.781 \\
AUC interpretation for & low & Moderate & Moderate \\
diagnostic accuracy & & 12 & 7 \\
OR & 4 & &
\end{tabular}

PPV: positive predictive value; NPV: negative predictive value; AUC: area under the curve; OR: odds ratio; PDP: Pervasive Developmental Problems.

describes the true positive rate, at the expense of the specificity, the true negative rate. The reason for this decision is the requirement to identify children with a risk of ASD by the CBCL $1.5-5$ as a level 1 screening instrument, not to make an accurate ASD diagnosis. The two scales PDP and Withdrawn showed a high sensitivity, which will result in few under-identifying or false negatives. At the cutoff point of $\mathrm{T}=64.5$ on the PDP scale, $83 \%$ of the children are correctly identified by the CBCL $1.5-5$. On the Withdrawn scale, $88 \%$ of the children with a $\mathrm{T}=60.5$ are truly at risk for an ASD diagnosis. Nevertheless, both scales demonstrate a lower specificity than required and present a risk of over-identifying ASD in children with OPD. Overidentification is often associated with the cost of unnecessary testing and produces concern for parents (Meisels, 1989). It is extremely worth emphasizing that children with high values on the CBCL $1.5-5$ and consequently at risk for an ASD diagnosis do not receive a final diagnosis. This is possible only through examination by an experienced child psychiatrist or psychologist with expertise in autism. The CBCL $1.5-5$ should point the way and reduce the current time lag of the ASD diagnostic. In addition, the false positive tested children show behavior problems that need further analysis, so they could benefit from a specialized assessment followed by early intervention. This is why we can accept a low specificity. In addition, it is important to mention that under-identification can result in a lack of awareness and also create confusion and anger. Disadvantages of under-identification are associated with more serious consequences than over-identification.

It is important for both scales, PDP and Withdrawn, to show a high AUC and indicate a moderate diagnostic accuracy as an essential measure.

By calculating the OR, we can interpret that the risk of having an ASD at $\mathrm{T}=64.5$ on the PDP scale is 7 times higher; at $\mathrm{T}=60.5$ on the Withdrawn scale, the risk is 12 times more frequent. In the logistic regression analysis, the scale Total Problems also indicated a significant predictive value of a risk for an ASD diagnosis. Nevertheless, we are not recommending the use of this scale for identification of children with a risk of an ASD by the CBCL 1.5-5 for the following reasons: In the ROC analysis, the Total Problems scale shows an unacceptably low diagnostic accuracy with a low AUC. Moreover, the scale Total Problems is a very unspecific scale and includes all 100 problem items of the CBCL 1.5-5. It is the sum of all scores. We can merely conclude that children with an ASD have in general more problems than OPD children.

Findings from this study are consistent with previous research. In these studies, two scales-PDP and Withdrawn - are also indicated as useful screening tools, despite a higher sensitivity than specificity (Muratori et al., 2011; Narzisi et al., 2013; Rescorla et al., 2014; Sikora et al., 2008). Similar cutoff points are calculated (Muratori et al., 2011; Rescorla et al., 2014).

Compared to our results, Muratori et al. (2011) describe the scale attention problems in the logistic regression analysis as a good predictor of an ASD presence. A reason for the research difference is most likely the lower mean age and age range of the ASD children in Muratori et al.'s (2011) study (average 44 months, ranging 24-60 months, compared to average 53.8 months, ranging $25-71$ months in this study).

In contrast to our findings, Havdahl et al. (2015) and Myers et al. (2014) indicated limited usefulness of the CBCL 1.5-5 for screening purpose because of a low AUC and a poor sensitivity and specificity compromise on the Withdrawn (AUC $=0.69$ or 0.752) and PDP scales (AUC $=0.68$ or 0.713 ). With our study, we could not confirm these findings for the German version of the CBCL 1.5-5.

The most significant limitation of this study, similar to the research already mentioned, is the low number of participants, which probably does not reflect the huge heterogeneity of the ASD and OPDs. There are also fewer children in the ASD group than in the OPD group. But due to the fact that children with an ASD were diagnosed in Germany at a mean age of 76 months (Noterdaeme and HutzelmeyerNickels, 2010) and only a limited number of children before this age will be seen by a psychiatrist with expertise in autism, the number of participants is quite acceptable. Missing data - relating to the procedure of completion of the CBCL 1.5-5-limits the power of this study. The results 
may have been influenced by whether the respondent completed the items of the questionnaire before or after knowing the child's final diagnoses. Most of the CBCL's 1.5-5 in this study were completed during the diagnosis process. An additional limitation of the study is that the different cognitive functioning of the children was not considered. Future studies are needed to examine the influence of the level of intelligence on the CBCL scales and cutoff points.

A strength of this study compared to previous research (Muratori et al., 2011; Myers et al., 2014; Sikora et al., 2008 ) is that there are no significant differences in either gender or age among the two groups. In our study, the percentage of males is also higher in the ASD group than in the OPD group. This refers to a clinical study and corresponds to the epidemiological distribution of a strong male prevalence of children with ASD (APA, 2013).

\section{Conclusion}

This study confirms the utility of the German version of the CBCL $1.5-5$ as a screening tool to identify children with a risk of ASD. The scales Withdrawn and PDP are especially suitable, although a risk of over-identifying should be considered. In conclusion, the CBCL $1.5-5$ can complement the pediatric examination as a quick and costeffective parent questionnaire. If the CBCL 1.5-5 shows increased values on the PDP and Withdrawn scale, the pediatrician should refer the child to a child psychiatrist with expertise in autism for a deeper evaluation. This could reduce the time lag between initial parental concern and an ASD diagnosis. This offers children an important opportunity for early and specific therapeutic intervention and subsequently an improved prognosis.

\section{Declaration of Conflicting Interests}

The author(s) declared no potential conflicts of interest with respect to the research, authorship, and/or publication of this article.

\section{Funding}

The author(s) received no financial support for the research, authorship, and/or publication of this article.

\section{References}

Achenbach TM and Rescorla LA (2000) Manual for the ASEBA Preschool Forms \& Profiles: An Integrated System of MultiInformant Assessment (Child Behavior Checklist for Ages 1 1 $12-$ 5; Language Development Survey; Caregiver-Teacher Report Form). Burlington, VT: University of Vermont.

American Psychiatric Association (APA) (2013) Diagnostic and Statistical Manual of Mental Disorders: DSM-5. 5th ed. Washington, DC: APA.

Dawson G, Jones Emily JH, Merkle K, et al. (2012) Early behavioral intervention is associated with normalized brain activity in young children with autism. Journal of the American Academy of Child and Adolescent Psychiatry 51(11): 1150-1159.
Freitag CM and Petermann F (2014) Autismus-SpektrumStörungen. Kindheit und Entwicklung 23(1): 1-4.

García-Primo P, Hellendoorn A, Charman T, et al. (2014) Screening for autism spectrum disorders: state of the art in Europe. European Child \& Adolescent Psychiatry 23(11): 1005-1021.

Havdahl KA, von Tetzchner S, Huerta M, et al. (2015) Utility of the Child Behavior Checklist as a Screener for Autism Spectrum Disorder. Autism Research 9: 33-42.

Ivanova MY, Achenbach TM, Rescorla LA, et al. (2010) Preschool psychopathology reported by parents in 23 societies: testing the seven-syndrome model of the child behavior checklist for ages 1.5-5. Journal of the American Academy of Child and Adolescent Psychiatry 49(12): 1215-1224.

Lord C, Rutter M, DiLavore PC, et al. (2012) Autism Diagnostic Observation Schedule, Second Edition (ADOS-2). Torrance, CA: Western Psychological Services.

Meisels SJ (1989) Can developmental screening tests identify children who are developmentally at risk? Pediatrics 83(4): 578-585.

Muratori F, Narzisi A, Tancredi R, et al. (2011) The CBCL 1.5-5 and the identification of preschoolers with autism in Italy. Epidemiology and Psychiatric Sciences 20(04): 329-338.

Myers CL, Gross AD and McReynolds BM (2014) Broadband behavior rating scales as screeners for autism? Journal of Autism and Developmental Disorders 44(6): 1403-1413.

Narzisi A, Calderoni S, Maestro S, et al. (2013) Child Behavior Check List $1 \frac{1 / 2-5}{2}$ as a tool to identify toddlers with autism spectrum disorders: a case-control study. Research in Developmental Disabilities 34(4): 1179-1189.

National Collaborating Centre for Women's and Children's Health (UK) (2011) Autism: recognition, referral and diagnosis of children and young people on the autism spectrum. Available at: https://www.nice.org.uk/guidance/cg128/documents (accessed 17 September 2015).

Noterdaeme M and Hutzelmeyer-Nickels A (2010) Early symptoms and recognition of pervasive developmental disorders in Germany. Autism the International Journal of Research and Practice 14(6): 575-588.

Pandolfi V, Magyar CI and Dill CA (2009) Confirmatory factor analysis of the child behavior checklist $1.5-5$ in a sample of children with autism spectrum disorders. Journal of Autism and Developmental Disorders 39(7): 986-995.

Rescorla L (1988) Cluster analytic identification of autistic preschoolers. Journal of Autism and Developmental Disorders 18(4): 475-492.

Rescorla L, Kim YA and Oh KJ (2014) Screening for ASD with the Korean $\mathrm{CBCL} / 1 \frac{1}{2}-5$. Journal of Autism and Developmental Disorders. Epub ahead of print 20 September. DOI: 10.1007/s10803-014-2255-y.

Rutter M, Le Conteur A and Lord C (2003) Autism Diagnostic Interview-Revised (ADI-R). Los Angeles, CA: Western Psychological Services.

Sikora DM, Hall TA, Hartley SL, et al. (2008) Does parent report of behavior differ across ADOS-G classifications: analysis of scores from the CBCL and GARS. Journal of Autism and Developmental Disorders 38(3): 440-448.

Swets JA (1988) Measuring the accuracy of diagnostic systems. Science 240(4857): 1285-1293.

Wiberg A, Heidenreich E, Springer S, et al. (2007) Belastung und Lebensqualität von Familien mit autistischen Kindern und Jugendlichen. Psychiatrische Praxis 34(S1): 66-68. 